\title{
Finite Density QCD with Wilson Fermions
}

\author{
Atsushi Nakamura* \\ Research Institute for Information Science and Education (RIISE), Hiroshima University, 1-7-1 \\ Kagamiyama, Higashi-Hiroshima 739-8521, Japan \\ E-mail: nakamura@rise.hiroshima-u.ac.jp \\ M. Hamada \\ Department of Physics, Kyushu University,6-10-1 Hakozaki, Higashi-ku, Fukuoka, 812-8581, \\ Japan \\ E-mail: hamada@phys.kyushu-u.ac.jp

\section{S. Motoki} \\ Graduate School of Biosphere Science, Hiroshima University 1-4-4 Kagamiyama, \\ Higashi-Hiroshima 739-8528, JAPAN \\ E-mail: motoki-shinji@hiroshima-u.ac.jp
}

\section{Takuya Saito}

Integrated Information Center, Kochi University, Kochi, 780-8520, Japan

\section{T. Takaishi}

Hiroshima University of Economics, Hiroshima 731-0192, JAPAN

E-mail: takaishi@hiroshima-u.ac.jp

\begin{abstract}
We report our on-going finite density and temperature project, where we employ Wilson fermions with the clover terms and Iwasaki improved gauge action. We are studying both pure imaginary and pure real chemical potential regions at the same time. For the real baryon chemical potential, we use the reweighting method. Since our goal is to simulate QCD at extreme conditions towards realistic parameters, i.e., lighter quark and smaller lattice spacing, and higher orders of fermion reweighting factors are non-negligible and converges slowly as the quark mass decreases, we develop a new simple Taylor expansion method, where an expansion parameter is $\exp ( \pm \mu / T)-1$, not $\mu$ itself. This gives simple formula for any order of fermion reweighting factors. We use also the hybrid-list and dilution techniques in "practical all-to-all propagators".
\end{abstract}

The XXVI International Symposium on Lattice Field Theory

July 14-192008

Williamsburg, Virginia, USA

\footnotetext{
*Speaker.
} 


\section{Introduction}

Although the numerical simulation of QCD at finite baryon density is one of the most difficult problems because of the sign problem in the fermion measure, there have been steady progresses in the finite density lattice QCD in these years. This is because the field is so attractive and challenging. Once we can study non-perturbatively QCD in the density- temperature plane, QCD will reveal its rich structure, and we expect to get experimental informations from high-energy heavy ion collisions, and astrophysical observations.

On the other hand, progresses of the simulation technique of lattice QCD are significant, and they will make it possible to perform realistic lattice QCD simulations.

We develop a HMC simulation code of Wilson fermions with the clover term, and the renormalization improved gauge action. This is currently practically best for analyzing QCD on a reasonably powerful machines. We employ this code for finite density and temperature QCD simulations. The fermion matrix has the form,

$$
\begin{aligned}
D(\mu) & =I-\kappa \sum_{i=1}^{3}\left[\left(1-\gamma_{i}\right) U_{i}+\left(1+\gamma_{i}\right) U_{i}^{\dagger}\right]-\kappa\left[\left(1-\gamma_{4}\right) e^{+\mu} U_{4}+\left(1+\gamma_{4}\right) e^{-\mu} U_{r}^{\dagger}\right] \\
& +(\text { Clover Term }) .
\end{aligned}
$$

Here we drop the site dependence.

The path integral after integrating the fermion Grassmann numbers is

$$
Z=\int \mathscr{D} U(\operatorname{det} D)^{N_{f}} e^{-S_{G}}
$$

We consider the two-flavor case, $N_{f}=2$.

The fermion matrix, Eq.(1.1), satisfies the flowing relation:

$$
D(\mu)^{\dagger}=\gamma_{5} D\left(-\mu^{*}\right) \gamma_{5}
$$

We study both the real and imaginary chemical potential regions at the same time, and try to extract informations on finite density aspects of QCD as much as possible.

For the real chemical potential, we use the reweighting technique. When the quark mass is decreasing and reaches to the realistic one, we need higher and higher terms of the fermion reweighting factor. This brings us two problems: the formula is more complicated and the stochastic estimation of higher reweighting factors becomes converges slowly. We propose a new Taylor expansion technique and an improvement of the stochastic estimation.

\section{Formulation}

\subsection{New Taylor expansion for reweighting}

Let us divide the fermion matrix, Eq.(1.1), into the one at $\mu=0$ and the rest,

$$
D(\mu)=D(0)+\Delta D(\mu) .
$$

$\Delta D$ consists of two parts,

$$
\Delta D=\Delta D_{+}+\Delta D_{-}
$$


where

$$
\begin{aligned}
& \Delta D_{+} \equiv-\kappa\left(e^{+\mu / T}-1\right)\left(1-\gamma_{4}\right) U_{4} \delta_{x_{4}, N_{t}}, \\
& \Delta D_{-} \equiv-\kappa\left(e^{-\mu / T}-1\right)\left(1+\gamma_{4}\right) U_{4}^{\dagger} \delta_{x_{4}, N_{t}} .
\end{aligned}
$$

Then,

$$
\begin{aligned}
\operatorname{det} D(\mu) & =\operatorname{det}\left(D_{0}+\Delta D\right)=\operatorname{det} D_{0} \operatorname{det}\left(1+D_{0}^{-1} \Delta D\right) \\
& =\operatorname{det} D_{0} \times \exp \left\{\operatorname{Tr} \log \left(1+D_{0}^{-1} \Delta D\right)\right\}=\operatorname{det} D_{0} \times e^{-\Delta S_{F} / N_{f}},
\end{aligned}
$$

where $D_{0} \equiv D(0)$ and

$$
\Delta S_{F} \equiv N_{f} \operatorname{Tr} \sum_{n=1} \frac{1}{n}(-1)^{n-1} \operatorname{Tr}\left[D_{0}^{-1}\left(\Delta D_{+}+\Delta D_{-}\right)\right]^{n}
$$

This $\Delta S_{F}$ is a fermion reweighting factor, and its $\mu$ dependence in each term can be factored out,

$$
\left(\Delta D_{+}\right)^{k}\left(\Delta D_{-}\right)^{l}=\left(e^{+\mu / T}-1\right)^{k}\left(e^{-\mu / T}-1\right)^{l} \times(\mu \text {-independent term })
$$

$D_{0}$ has no $\mu$ dependence, and, once can calculate a reweighting factor, we can construct reweighting factors for any value of $\mu / T$ simultaneously for each configuration.

For quark propagators, which we need to construct hadron propagators, we can expand in a similar manner,

$$
D^{-1}=\left(D_{0}+\Delta D\right)^{-1}=\left(1+D_{0}^{-1} \Delta D\right)^{-1} D_{0}^{-1}=\sum_{n=0}\left(-D_{0}^{-1} \Delta D\right)^{n} D_{0}^{-1} .
$$

We plan to use this formula for hadron screening mass calculations at finite density, because this is much simpler than the present Taylor expansion method[4].

\subsection{Practical all to all propagators}

\section{Hybrid lists}

Now main calculation for evaluating the fermionic reweighting factor is

$$
\operatorname{Tr}\left(D_{0}^{-1} \Delta D\right)^{n}
$$

Recently TriLat group proposed an effective way to calculate all-to-all propagators[2]. We borrow their idea to calculate Eq.(2.8).

Suppose we have a series of vectors $\left|\phi_{k}\right\rangle$ which are complete,

$$
\sum_{k}\left|\phi_{k}\right\rangle\left\langle\phi_{k}\right|=I
$$

There are many (theoretically infinite) choices of such vectors. One is eigen values of the matrix $Q \equiv \gamma_{5} D_{0}$,

$$
Q\left|\lambda_{k}\right\rangle=\lambda_{k}\left|\lambda_{k}\right\rangle
$$


In order to satisfy Eq.(2.9), we require the following relations:

$$
\left\langle i\left|\left(\sum_{k}\left|\phi_{k}\right\rangle\left\langle\phi_{k}\right|\right)\right| j\right\rangle=\delta_{i, j} .
$$

So-called noise vectors fulfill the condition. TriLatt group shows that an effective way is to use truncated eigen vectors, and to compensate them by the noise vectors.

Let us introduce a projection operator, $P=1-\sum_{1}^{N_{e v}}\left|\lambda_{k}\right\rangle\left\langle\lambda_{k}\right|$. We calculate $Q^{-1}(1-P)$ by $\sum_{1}^{N_{e v}}\left|\lambda_{k}\right\rangle\left\langle\lambda_{k}\right|$, and estimate $Q^{-1} P$ by noise vectors. Using $Q^{-1}=Q^{-1}(1-P)+Q^{-1} P$,

$$
Q^{-1}=Q^{-1} \sum_{k}\left|\phi_{k}\right\rangle\left\langle\phi_{k}\left|=\sum_{k=1}^{N_{e v}} \frac{1}{\lambda_{k}}\right| \lambda_{k}\right\rangle\left\langle\lambda_{k}\left|+\frac{1}{N_{\text {noise }}} \sum_{k=N_{e v}+1}^{N_{e v}+N_{\text {noise }}}\right| \xi_{k}\right\rangle\left\langle P \eta_{k}\right|
$$

where $\eta$ are noise vectors, and $\xi$ is a solution of $Q \xi_{k}=P \eta_{k}$.

Using $Q^{-1}=\sum\left|u_{k}\right\rangle\left\langle w_{k}\right|$ and $D_{0}^{-1}=Q^{-1} \gamma_{5}$, we can evaluate each term in the fermion reweighting factor as

$$
\begin{array}{r}
\operatorname{Tr}\left(D_{0}^{-1} \Delta D\right)^{n}=\sum_{k} \operatorname{Tr}\left|u_{k}\right\rangle\left\langle w_{k}\right| \gamma_{5}\left(D_{0}^{-1} \Delta D\right)^{n-1} \\
=\sum_{k}\left\langle w_{k}\left|\gamma_{5} D_{0}^{-1} \Delta D \cdots D_{0}^{-1} \Delta D\right| u_{k}\right\rangle .
\end{array}
$$

\section{Dilution}

For the vector $\phi_{k}$, we may adopt a unit vector, $\phi_{k}(i)=\delta_{i, k}$, where $i$ and $k$ are $i=(a, \alpha, x), k=$ $(b, \beta, y)\left(a, b:\right.$ color, $\alpha, \beta$ : Dirac, $x, y:$ site). In this case, we get the exact elements of $Q^{-1}$, but we must solve the linear equation $N_{c} \times 4 \times$ Lattice Volume times. The random noise vectors are another extreme.

TriLat collaboration suggests a compromise. We introduce noise vectors, $\eta^{(a, \alpha)}(x) \equiv \vec{e}^{a} \vec{e}^{\alpha} \chi(x)$, where $\vec{e}^{a}$ and $\vec{e}^{\alpha}$ are unit vector in color and Dirac space, and $\chi(x)$ are random numbers which satisfy $\operatorname{Ave}(\chi(x) \chi(y))=\delta_{x, y}$.

\subsection{Imaginary chemical potential}

In case of pure imaginary chemical potential, $\mu=i \mu_{I}$, the relation, Eq.(1.3), becomes,

$$
D\left(\mu_{I}\right)^{\dagger}=\gamma_{5} D\left(\mu_{I}\right) \gamma_{5}
$$

and there is no sign problem, i.e., $\operatorname{det} D$ is real.

\section{Numerical Test}

We will show some preliminary data to see if such simulations work. The lattice size is $8^{3} \times 4$. In Fig.1, we study the contributions from the first and the second term of Eq.(2.12) for $\operatorname{Tr} D_{0}^{-1} \Delta D$. $\beta=1.85$ which corresponds to $T / T_{c}=0.99$ [1]. The hopping parameter, $\kappa$, is 0.140070 . First we suppress the noise term, i.e., $N_{\text {noise }}=0$, and increase $N_{e v}$. Until $N_{e v}=10$ the real part of the obtained $\operatorname{Tr} D_{0}^{-1} \Delta D$ changes rapidly. Then we increase $N_{\text {noise }}$ from 1 to 5 . The change is less. 
Although we are still at a very preliminary stage, this suggests that the contribution from the eigen vectors is large, and we need still large number of the noise vectors.

For the imaginary chemical potential, we set $\beta=1.90$, which corresponds $T / T_{c}=1.08$. The hopping parameter is $\kappa=0.138817$. We show in Fig.2. Real part of Polyakov lines as a function of the pure imaginary chemical potential, $\mu_{I}$. Because of the Roberge-Weiss symmetry[5], any obserbables in the pure imaginary chemical potential has the periodicity, $2 \pi / N_{c}$. We see here just the first period.

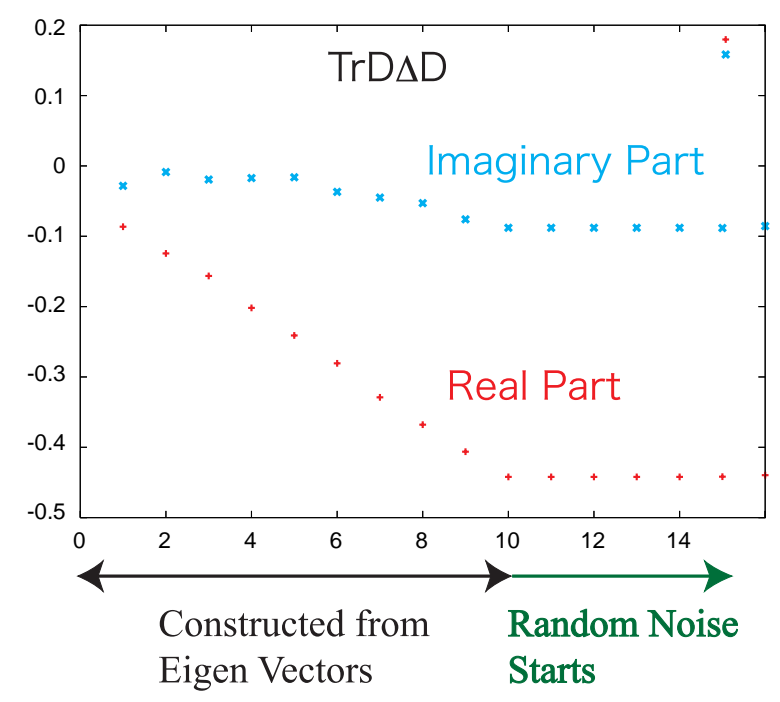

Figure 1: Contributions of the first term (Eigen functions) and the second term (Noise vectors) in the right hand side of Eq.(2.12) to $\operatorname{Tr} D_{0}^{-1} \Delta D$.

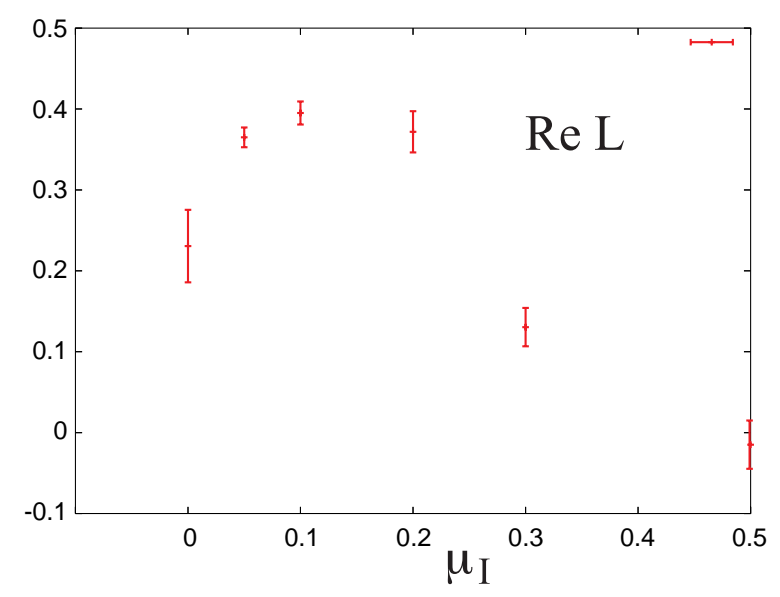

Figure 2:

\section{Summary}

We have just started to check the validity of the algorithms and lattice actions. The numerical 
tests presented here are still not very convincing, and we must see behaviors with much less quark masses.

If the algorithm and code works well there, we can investigate many interesting observables. First we can provide predictions at finite imaginary chemical potentials. Then one can compare them with those by PNJL model by tuning its parameters[6]. Then the PNJL model has much reliable prediction power at the real baryonic chemical potentials.

Note that the expansion with respect to $e^{ \pm \mu / T}-1$ can be applied also for the pure imaginary chemical potental. Although we can calculate at finite $\mu$ if $\mu$ is pure imaginary, we need a fitting ansatz for converting results to the real chemical potential. Present standard way to fit finite $\mu_{I}$ is polynomical functions of $\mu_{I}$, but it seems to have a limitation. It is worth while to try this new expansion formula.

Another interesting target is hadron screening masses at finite temperature. So far there have been such study only with the staggered fermions. There it becomes unclear which flavor and Dirac components we put the chemical potential. This problem is more complicated when we consider both baryonic and iso-spin chemical potential.

\section{Acknowledgement}

Numerical calculatins here were done by SR11000 at Hiroshima University, SX-8 at RCNP, Osaka university and SR11000 at KEK. The work is partially supported by Grant-in-Aid for Scientific Research by Monbu-kagakusyo, No. 17340080 and 20340055.

\section{References}

[1] WHOT-QCD Collaboration: Y. Maezawa et al, arXiv:hep-lat/0702004.

[2] J. Foley et al, Comput.Phys.Commun. 172 (2005) 145-162. (arXiv:hep-lat/0505023)

[3] S. Muroya et al., Prog. Theor. Phys. 110, (2003) pp.615-668 (hep-lat/0306031)

[4] QCD-TARO Collaboration: I. Pushkina et al., Phys. Lett. B609 (2005) 265-270, (hep-lat/0410017)

[5] A. Roberge and N. Weiss, Nucl. Phys. B275, 734 (1986).

[6] Y. Sakai et al., arXiv:0806.4799. 